\title{
BIO-ELECTRICAL IMPEDENCE PLETHYSMOGRAPHY- DESIGNING AN EFFICIENT NON-INVASIVE ELECTRODE FOR CARDIAC RATE ANALYSIS
}

\author{
Daniya Umer, Ghanwa Ellahi, Warda Ellahi, Saba Ayub \&. Zia Mohy Ud Din
}

1. Sir syed university of engineering and technology

Corresponding author: umer.daniya16@gmail.com

\begin{abstract}
Cardiac rate analysis is constituted as one of the most essential parts of physiological patient monitoring. In this research paper, it has been investigated that electrical impedance plethysmography is the most conducive technique for this purpose. It is perceptible that the artery closest to the epidermis will generate a precise reading and for that purpose, we have designed an efficient non-invasive model based on a tetraelectrode setup placed in contact with the stratum corneum of the skin. By varying electrode size and the distance between the electrodes we can efficiently judge the point where the electric field reaches the artery. The simulation of this model is carried out by Comsol Multiphysics.
\end{abstract}

\section{KEYWORDS}

Plethysmography, Electrical impedance plethysmography (EIP), electrode dimensions, non-invasive, stroke volume, seismocardiography, Photoplethysmography, Simulation, cardiovascular/pulse rate, Comsol Multiphysics.

\section{INTRODUCTION}

Cardiovascular rate basically deals with heartbeat and stroke volume. The heartbeat is the number of times the heart beats per minute whereas stroke volume deals with the volume of blood pumped into the ventricle of the heart during every beat. Fundamentally, the pulse rate is greater during a state of exercise as compared to the pulse rate at rest. It is not necessary for every healthy person's pulse rate to be the same. Normally, an adult's pulse rate varies between 60-100 BPM (beats per minute). Essentially, the cardiovascular rate indicates the physical wellness and fitness of a person and how different changes in the environment can affect that rate.There are a number of ways through which this rate can be determined. ECG, seismocardiography, Photo Plethysmography and Electrical Impedance Plethysmography. Seismocardiography deals with the recording of the vibrations produced through the myocardial wall. In 1990, a paper was published regarding this concept by Salemo, D.M and Zanetti $\mathrm{J}$ in the Journal of Cardiovascular Technology (Salemo, 1990). The drawback regarding this technique was the presence of motion artefacts produced due to the movement of the lungs. These days, the accurate yet most complex method is of Electrocardiography (ECG). The ECG consists of an assemblage of electrodes placed at specific points on the body. These electrodes pick signals from the surface of the body and give output in the form of a wave. This wave can be studied for the detection of heart rate. (The RR interval determines this) The greatest disadvantage of this method is that the process itself is arduous and time-consuming due to the arrangement of the different electrodes and can become very uncomfortable for patients who require continuous observation.Photoplethysmography is another technique by which heart rate can be detected. According to a paper published by John Allen in 2007 this method is simple and low-cost. The drawback is found to be in the motion artefacts caused by the slight movements of the hand, thus decreasing accuracy and also that a regulator/potentiometer is required for signal adjustment. (Allen, 2007).On the other hand, Electrical Impedance Plethysmography appears to be the most practical solution as compared to the above mentioned techniques of cardiovascular rate detection. EIP deals with the study of the electrical properties of tissues. A number of electrical methods have been employed to study the vascular phenomena and this technique has proved to be one of the best methods to determine the change in tissue volume from which cardiovascular rate can be determined based on the measurement of electrical impedance on the body surface. Blood vessels expand and contract with respect to the change in heart rate, hence change of blood volume with each heartbeat causes a change in the electrical impedance of the arteries. The output generated by EIP is done by producing an electric field at a specified intensity. It works on the basis of a tetra electrode setup in which the electrodes are placed at very small distances from each other. Due to the conciseness of this model, motion artefacts are negligible. A paper published in 2005 in the Journal of Medical and Biological Engineering and Computing (Kristiansen, 2005) showed the use of a hand held system based on EIP for heart rate detection. The difficulty regarding this system is that both the hands are used to take the reading, the system also looks very heavy and cannot be used for the continuous monitoring of heart rate, while, as shown in this paper, if a wrist or waist band is used (where the electrodes are attached underneath), the system becomes much easier to use. In another article which was published in 2012, shows a cuff measuring cardiovascular rate properties based on the principle of EIP has been used for the diagnosis of a specific disease. Since some patients require continuous monitoring of the heart rate for diagnosis, a cuff can tend become uncomfortable for prolonged periods of time. As previously mentioned, a wrist or waist band is the practical option (Hull, 1976).In this paper, EIP has been studied and analyzed using a Skin Model. We have used Comsol Multiphysics to build the model of the skin, along with its layers, showing the femoral artery. In this model we have adjusted the electrodes used according to their dimensions and distance between them to find out the best position where the cardiovascular rate can be observed.

\section{MATERIALS AND METHOD}

To study EIP, a skin model has been designed. The simulation of this model on software basis can easily be carried out by Comsol Multiphysics. Since projects related to human physiology prove to be arduous for study and analysis, the Comsol Multiphysics software becomes of utmost importance for Biomedical Engineers as these projects can be analyzed and understood with ease in a small time frame. Considering the fact that physiological systems are very complex in nature, we only considered the following parameters in the construction of our skin model: (1) Stratum Corneum, (2) Epidermis, (3) Dermis and (4) Muscle. 


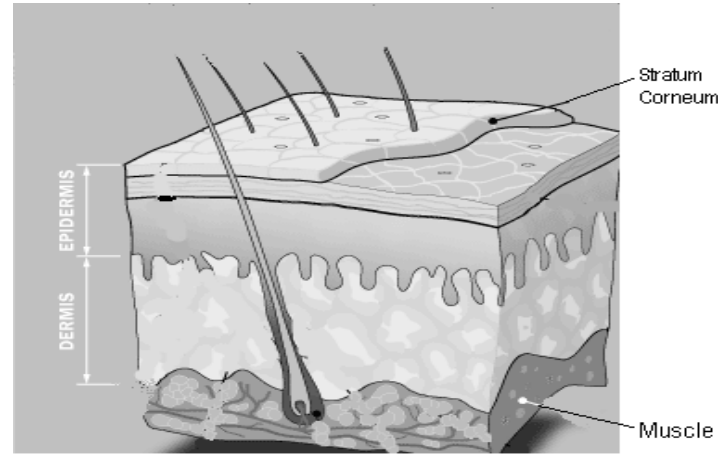

Fig.1 A cross-sectional area of the skin.

These parameters are set according to the conductivity and permittivity of each layer. Since the relative permittivity is constant, i.e. 1, the electrical conductivity of each layer is defined as follows.

\begin{tabular}{|cl|l|}
\hline \multicolumn{2}{|l|}{ LAYERS OF SKIN } & $\begin{array}{l}\text { ELECTRICAL } \\
\text { CONDUCTIVITY }\end{array}$ \\
\hline 1. & Stratum corneum & $0.0005 \mathrm{~S} / \mathrm{m}$ \\
\hline $2 . \quad$ Epidermis & $0.95 \mathrm{~S} / \mathrm{m}$ \\
\hline $3 . \quad$ Dermis & $0.2 \mathrm{~S} / \mathrm{m}$ \\
\hline $4 . \quad$ Muscle & $0.66 \mathrm{~S} / \mathrm{m}$ \\
\hline $5 . \quad$ Blood & $0.8 \mathrm{~S} / \mathrm{m}$ \\
\hline
\end{tabular}

Table 1: Electrical Conductivity of the layers of the skin and blood.

The first step was to perform an A-Scan ultrasound on the required area, i.e. the femoral artery. The result is shown below.

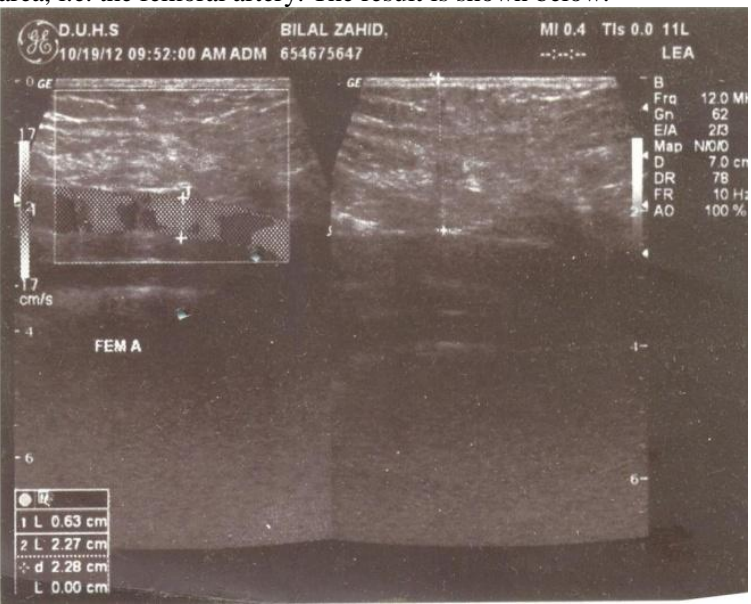

Fig 2: Ultrasound scan of the femoral artery.

A model on Comsol Multiphysics is made using the ultrasound result and is designed according to the conductivity and permittivity of each layer. The resultant model is shown below.

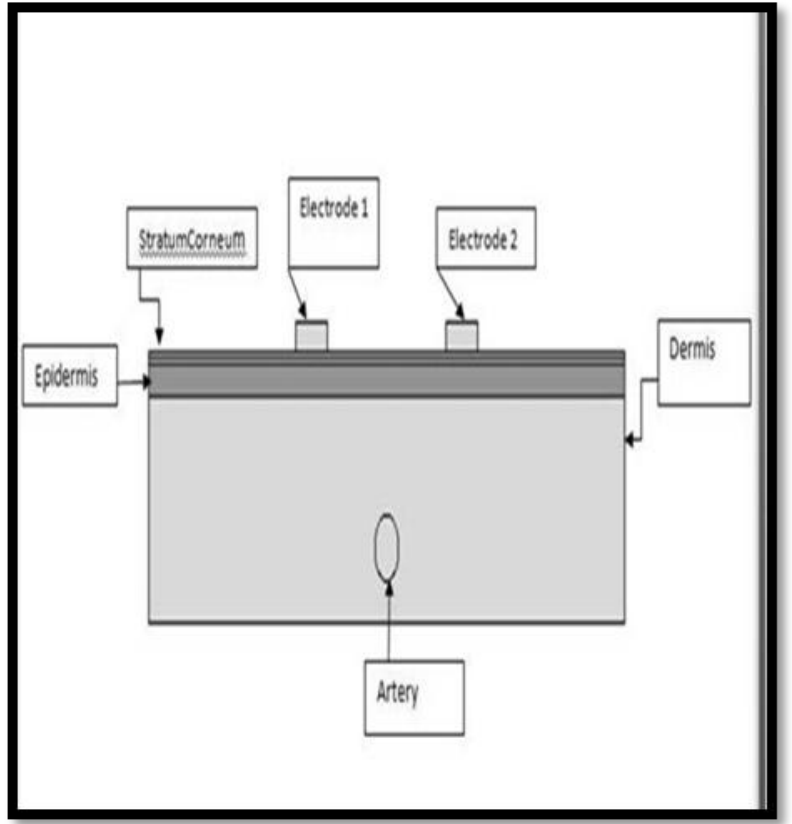

Fig 3: Initial model constructed in Comsol.

Comsol performs the simulation on the basis of Maxwell's Equation. This equation is explained as follows.

$\Delta V=\frac{\mathrm{gL}^{*}}{\mathrm{z}^{2}}\left(\Delta \mathrm{Z}_{\mathrm{g}}+\Delta \mathrm{Z}_{\mathrm{v}}\right)$

Here,

$\Delta V=$ change in arterial blood volume.

$\mathrm{L}=$ distance between the electrodes

$p=$ blood/artery's resistivity

$Z_{z}=$ basal impedance in absence of blood flow

$z_{0}=$ impedance due to change in blood resistivity

$z_{w}=$ impedance due to change in blood volume

\section{A. SKIN MODEL}

The skin model is designed according to the depth and diameter of the femoral artery shown in the ultrasound report. This artery is denoted by the circle shown in Fig 3.

Rectangles are used to design the electrodes which are placed on the surface of the skin. Table 3 shows the dimensions according to which the layers of the skin have been designed.

\begin{tabular}{|c|c|c|}
\hline LAYERS OF SKIN & HEIGHT (mm) & WIDTH (mm) \\
\hline $\begin{array}{ll}\text { A. } & \begin{array}{l}\text { Stratum } \\
\text { corneum }\end{array}\end{array}$ & 0.1 & 45 \\
\hline B. Epidermis & 0.25 & 45 \\
\hline C. Dermis & 1 & 45 \\
\hline D. Muscle & 30 & 45 \\
\hline
\end{tabular}




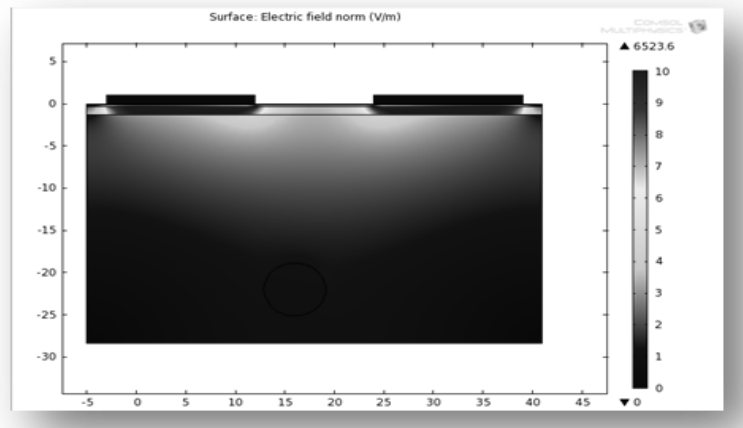

Fig 4: Output represented by a model showing the electrical intensity represented by rainbow colours.

\section{B. SIMULATION}

Simulation is basically the analyzing and studying the output of the model in imaginary world before it's implemented in the real world. So that we can design the electrodes efficiently, we've done two simulations in this research paper.

$>$ Simulation for inter-distance

$>$ Simulation for area

\section{SIMULATION FOR INTER-DISTANCE}

By changing the distance between the electrodes, the intensity was observed and the best result was found out to be $1.14 \mathrm{~V} / \mathrm{m}$ at $17 \mathrm{~mm}$.
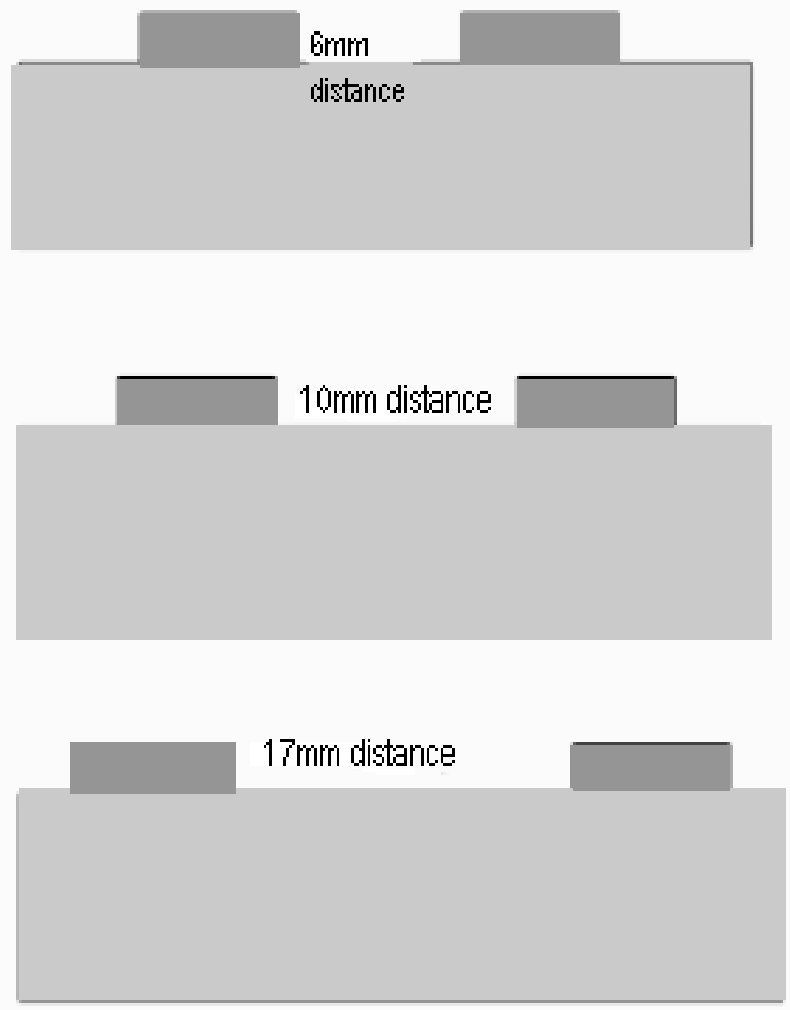

Fig 5: Representing the change of distance between electrodes. Simulation for area
By changing the area of the electrodes, we observed the intensity and the best result was found out to be $1.1434 \mathrm{~S} / \mathrm{m}$ at an area of $14 * 1$ $\mathrm{mm}^{\wedge} 2$.

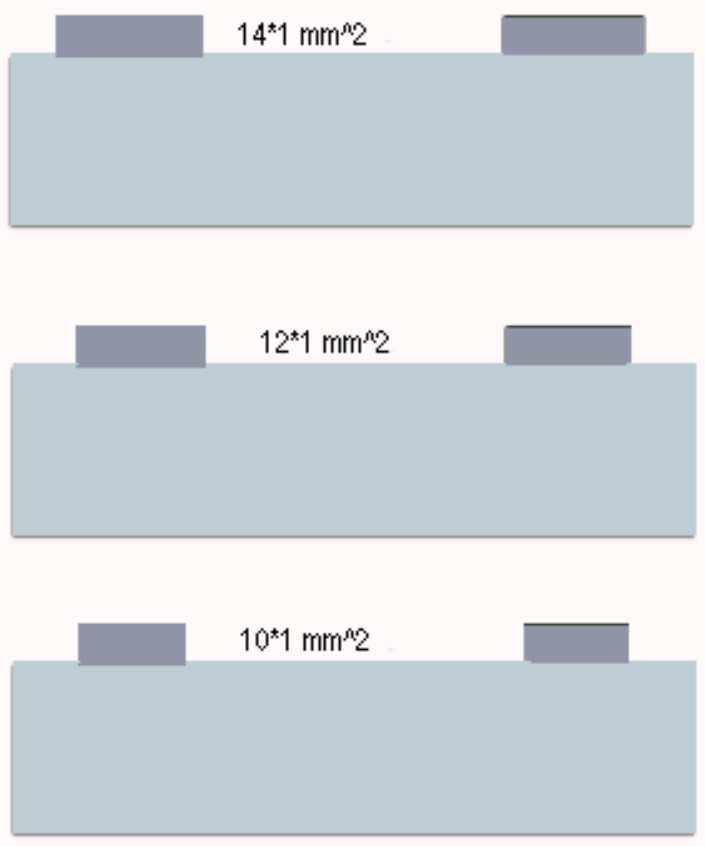

Fig 6: Representing change of area in the electrodes.

\section{RESULTS}

The final results for each simulation are as follows.

- Optimal inter-distance between electrodes: $17 \mathrm{~mm}$

- Optimal area of the electrodes: $14 \mathrm{~mm}$

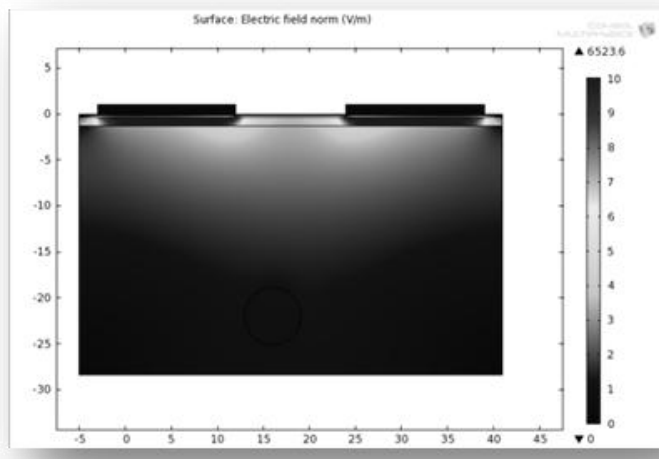

Fig 7: The final simulation result achieved.

The output of these simulations can be represented in a graphical form as well. 


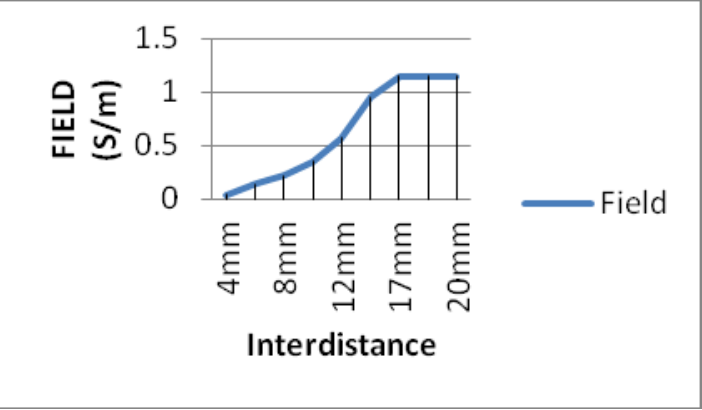

Fig. 8: Graph showing the relationship between inter-distance of electrodes and the field generated.

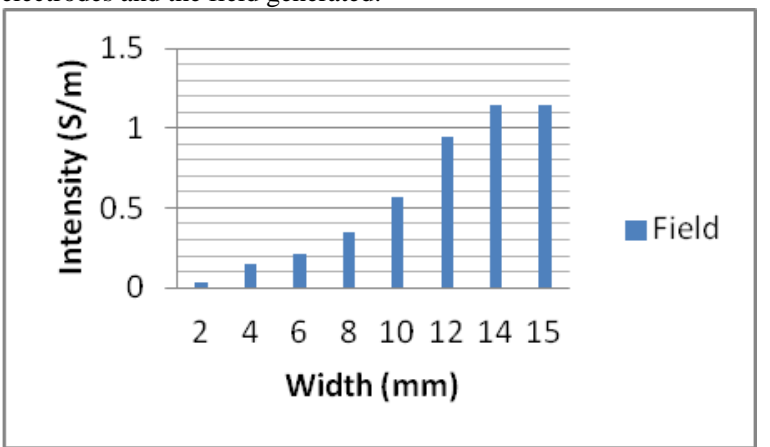

Fig 9: Graph showing the relationship between the width of electrodes and the field generated.

The best field of intensity, i.e. $1.1434 \mathrm{~S} / \mathrm{m}$, was observed at an interdistance of $17 \mathrm{~mm}$ and an area of $14^{*} 1 \mathrm{~mm}^{\wedge} 2$. This field has proven to be the best intensity required to stimulate the femoral artery without being hazardous to the femoral artery.

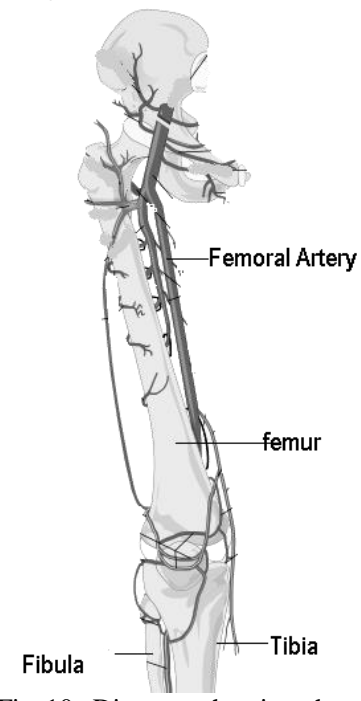

Fig 10: Diagram showing the position of the femoral artery in the upper leg.

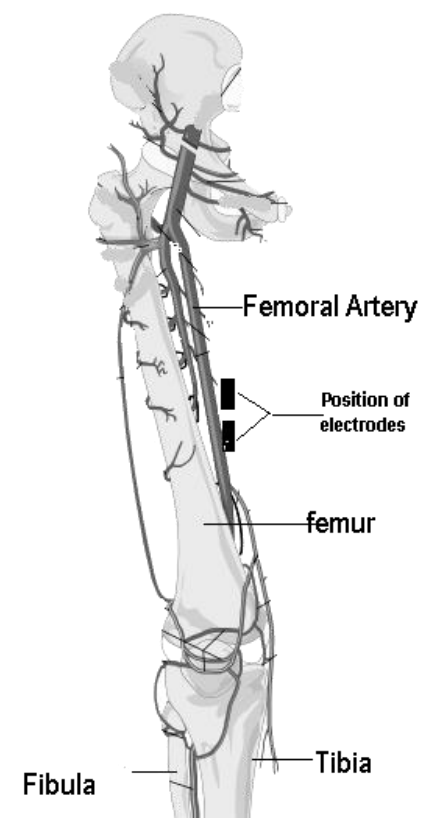

Fig 11: Positioning of the electrodes.

\section{CONCLUSION}

The electrodes designed in this paper prove to be an accurate example for the demonstration of electrical impedance plethysmography. After simulation of these electrodes with respect to inter-distance and area, an accurate value of electrical field intensity was computed. At this value, the femoral artery was stimulated without being hazardous. These electrodes can be used effectively for the measurement of cardiovascular rate non-invasively thus providing a useful application of Electrical Impedance Plethysmography.

\section{REFERENCES}

- Salemo, D.M., Zanetti (1990) Seismocardiography - a new technique for recording cardiac vibrations - concept, method, and initial observations $\mathrm{J}$ in the Journal of Cardiovascular Technology.

- Allen, J. (2007). Photoplethysmography and its application in clinical physiological measurement. Physiological measurement, 28(3), R1.

- Kristiansen, N. K., Fleischer, J., Jensen, M. S., Andersen, K. S., \& Nygaard, H. (2005). Design and evaluation of a handheld impedance plethysmograph for measuring heart rate variability. Medical and Biological Engineering and Computing, 43(4), 516-521.

- Hull, R., Van Aken, W. G., Hirsh, J., Gallus, A. S., Hoicka, G., Turpie, A. G., ... \& Gent, M. (1976). Impedance plethysmography using the occlusive cuff technique in the diagnosis of venous thrombosis. Circulation, 53(4), 696-700.

- Baumgartner, Chunlea, and Roche, 1988

- Kushner and Shoeller, 1986; Lukaski et al., 1985. Wikswo, J.P. (1975). "Non-invasive magnetic measurement of the electrical and mechanical activity of the heart. Stanford University, Stanford, Thesis, pp. 304. (Ph.D. thesis)"

- Geselowitz, D. B. (1971). An application of electrocardiographic lead theory to impedance plethysmography. Biomedical Engineering, IEEE Transactions on, (1), 38-41.

- "Department of Defense Modeling and Simulation the term as be defined(M\&S) Glossary", DoD 5000. 